\title{
La transformation hongroise : grévistes de la faim, syndicalistes, et membres du gouvernement
}

\section{Béla Greskovits}

\section{(2) OpenEdition}

1 Journals

\section{Édition électronique}

URL : http://journals.openedition.org/conflits/326

DOI : $10.4000 /$ conflits.326

ISSN : 1777-5345

Éditeur :

CCLS - Centre d'études sur les conflits lilberté et sécurité, L'Harmattan

\section{Édition imprimée}

Date de publication : 15 mai 1995

ISSN : 1157-996X

Référence électronique

Béla Greskovits, « La transformation hongroise : grévistes de la faim, syndicalistes, et membres du gouvernement », Cultures \& Conflits [En ligne], 17 | printemps 1995, mis en ligne le 15 mars 2006, consulté le 30 mars 2021. URL : http://journals.openedition.org/conflits/326 ; DOI : https://doi.org/ $10.4000 /$ conflits.326

Ce document a été généré automatiquement le 30 mars 2021.

Creative Commons License 


\title{
La transformation hongroise : grévistes de la faim, syndicalistes, et membres du gouvernement
}

\author{
Béla Greskovits
}

1 Une transition vers quoi ? ${ }^{1}$ Dans le tumulte des années 1989 et 90, nombre de politiciens, de politistes, d'économistes, d'experts partageaient l'idée que la chute du régime communiste en Europe de l'Est serait bientôt suivie d'une transition vers la démocratie libérale et l'économie de marché. Deux ans plus tard, les analystes devinrent moins optimistes quant à la consolidation de la démocratie. Alors que les économistes étaient frappés par la profondeur et la longueur de la récession, les sociologues et politistes signalèrent de plus en plus les carences structurelles et institutionnelles des sociétés post-communistes. Les pays en transition vers la démocratie manquaient de partis institutionnels, de leaders politiques confirmés, d'élites responsables, d'un appareil d'Etat capable et efficace, d'une société civile organisée, de classe moyenne et de capitalistes, pour ne citer que quelques éléments parmi une longue liste. S'il n'est pas très difficile de prouver l'inexistence de nombreux éléments constitutifs des démocraties occidentales, il ne faudrait cependant pas déduire de cette carence l'impossibilité d'un enracinement démocratique en Europe de l'Est, et ce quelle qu'en soit la forme. De plus, il convient de rester vigilant et de ne pas reproduire l'approche erronée, si vivement critiquée par Alexander Gerschenkron dans un contexte différent. Selon lui, ceux qui ont expliqué l'écart existant au XIXe siècle entre l'économie de la Grande Bretagne et celle du Continent par l'absence au sein de l'économie continentale des éléments fondamentaux de l'industrialisation, ont nécessairement occulté deux des vecteurs institutionnels de la révolution industrielle : les banques, qui ont fourni les investissements financiers à long terme, et l'Etat ${ }^{2}$. Il nous faut aussi prendre en compte l'avertissement de Guillermo O'Donnell pour comprendre le développement démocratique. En effet, on doit éviter de caractériser les systèmes politiques en «indiquant les attributs qui leur font défaut (la représentativité, l'institutionnalisation, par exemple), et en décrivant les aléas qu'ils ont pu connaître sur le plan politique et économique" car cette approche "risque d'induire une 
téléologie empêchant une conceptualisation appropriée des différents types de démocratie émergents" ${ }^{13}$. Cette critique comme le thème esquissé précédemment semble être valide et pertinente bien au-delà du contexte latino-américain. Voilà pourquoi il nous semble que la science politique peut aller plus loin dans l'analyse en expérimentant de nouveaux concepts sur "les sous-espèces démocratiques", "les démocraties possibles", "les types de démocratie" dans le cas des transitions démocratiques en Europe de l'Est, plutôt que d'enterrer de façon hâtive la notion de «transformation démocratique » post-communiste, sous prétexte qu'il lui manquerait quelques ingrédients de la recette occidentale ${ }^{4}$. La conceptualisation appropriée de ce qui a émergé dans la politique et l'économie d'Europe centrale constituait le sujet même de notre recherche lorsque nous avons élaboré notre projet intitulé «transition vers quoi? " au département de science politique à l'université d'Europe centrale de Budapest. En menant notre recherche nous nous sommes rendus compte combien il était difficile de repérer le chemin du développement démocratique dans les changements rapides et désordonnés du système. Conscients des difficultés liées à la fluidité de ce dernier, notre but est ici de mieux comprendre la nature de la démocratie qui émerge actuellement en Hongrie, et de contribuer peut-être à sa conceptualisation par quelques réflexions. Pour mieux connaître les processus de démocratisation non encore achevés ni consolidés, la meilleure démarche à adopter est d'observer et d'étudier les agents démocratiques lorsqu'ils agissent, luttent et s'opposent dans le contexte global du système politique institutionnel. Il existe des moments spécifiques comme les élections ou les conflits sociopolitiques graves - où les actions et les événements se compliquent, où l'arène politique "s'échauffe", où les acteurs politiques font face à des défis importants. Leurs réponses peuvent être alors révélatrices, beaucoup plus qu'en période ordinaire, de leurs buts, de leurs ressources et de leurs stratégies, mais aussi de l'adaptation, de l'élasticité et de la durabilité du système lui même. Les pactes sociaux constituent assurément, en ce sens, des moments significatifs. Par conséquent, pour introduire la démocratisation hongroise «en cours de réalisation ", nous avons choisi l'exemple du pacte social de 1992, auquel on peut joindre les événements politiques qui l'ont précédé et suivi. Notre analyse est en partie basée sur une couverture complète des événements qui ont eu lieu entre octobre 1992 et janvier 1993, fournie par les quotidiens influents Magyar Hirlap et Népszabadság, sur d'autres études portant sur le même cas et enfin sur des entretiens privés menés avec les artisans et les partisans du pacte social. Nous présenterons dans un premier temps, une vue générale de la situation économique, et du contexte de la politique économique dans lequel le gouvernement optait pour un programme d'austérité à l'automne 1992. Nous analyserons ensuite le conflit socio-politique conséquence du mécontentement issu de l'épreuve sociale liée aux mesures d'austérité. Nous identifierons différentes menaces qui jouent contre les réformes prévues, à savoir les moyens économiques et politiques que les différents groupes économiques essayent d'utiliser pour arrêter ou modifier les politiques d'ajustement. Nous distinguerons également les organisations que l'on retrouve derrière les protestations et évaluerons leur force organisationnelle et leur influence politique. Nous aborderons le pacte social tripartite, signé le 21 novembre 1992. L'analyse mettra l'accent sur l'identification des différents intérêts du gouvernement, des syndicats et du monde des affaires, chacun souhaitant faire aboutir une solution négociée, et la façon dont le pacte a refaçonné les politiques initiales et les mesures de compensations. On tentera également de montrer qui sont les gagnants et les perdants du pacte social. Enfin, on évaluera les lendemains du pacte, notamment les 
réactions et les stratégies des vainqueurs et des vaincus. Nous commenterons dans une seconde partie quelques uns des résultats des recherches sur la pactes sociaux publiés dans la «littérature ». Nous réfléchirons ensuite sur "la typologie des démocraties", élaborée récemment dans le contexte latino-américain par Carlos $\mathrm{H}$. Acuna et William C. Smith, en prêtant une attention toute particulière aux traits caractéristiques du système démocratique hongrois tel qu'il a été révélé par le conflit socio-politique et sa résolution. En effet, on tentera de voir si cette typologie peut nous apporter des éléments significatifs sur la démocratisation hongroise, et si inversement quelques uns des points spécifiques de cette démocratisation peuvent servir à verifier la typologie et peut-être l'enrichir empiriquement.

2 Le pacte social hongrois de novembre 1992 La situation économique globale et le contexte politique 1992 fut l'une des pires années de l'histoire économique postcommuniste hongroise. Les indicateurs macro-économiques laissaient apparaître alors pour la troisième année consécutive, un paysage économique dévasté : une profonde récession continue, une inflation galopante, des investissements en chute, une hausse importante du chômage, une paupérisation rapide et un déficit budgétaire record ainsi que des dégradations sociétales liées à cette transformation. Le pire était pourtant l'absence de signe d'amélioration de la situation. A l'évidence, l'économie hongroise était bien loin de "reprendre du souffle" (voir tableau 1 ci-dessous)

Sélection d'indicateurs économiques :

4 GDP Investissements Taux d'inflation Taux de chômage Balance du Budget Balance des paiements courants (Millions \$) 19890.97170 .58 -3.1 1990 -4 -7 291.7 - $1271991-11$-12

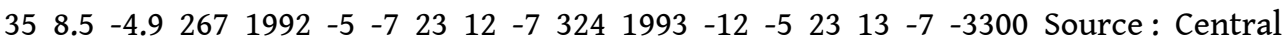
Statistical Office ; Magyar Hírlap, Heti Világgazdaság

5 Le climat politique était également tendu. En effet, depuis août 1992 le Forum Démocratique Hongrois (MDF), le principal parti de coalition, suite à un pamphlet politique de l'un de ses vice-présidents : István Csurka, était confronté à une division qui allait grandissante. En outre, les manifestations de masse de l'aile droite et des sociaux-libéraux, les attaques répétées provenant d'extrémistes et du gouvernement contre les médias "anti-gouvernementaux" et les provocations fascistoïdes contre le Président de la Hongrie témoignaient d'une atmosphère politique explosive en septembre et octobre. Ajouté à cela, Csurka et ses partisans manifestaient leur volonté avec une insistance toujours plus grande pour des changements fondamentaux dans la politique gouvernementale, et le limogeage des "conspirateurs" (comme la "mafia financière internationale" menée par le FMI, la Banque mondiale, et ses "agents internes"), censés avoir allourdi les programmes macro-économiques d'austérité, entraînant une détérioration excessive du niveau de vie ${ }^{5}$. Des technocrates réformistes, proches du ministre des Finances, Mihály Kupa, étaient de plus en plus ouvertement attaqués par les extrémistes du M.D.F., affirmant que la situation dans le pays ne s'améliorerait pas avant qu'on ait nettoyé le Ministère des Finances de ses "économistes" et "experts financiers à l'esprit étroit", pour les remplacer par des "penseurs stratégiques nationaux". Dès lors, le Ministre des Finances et son équipe avaient toutes les raisons de se sentir affaiblis et menacés. Par ailleurs, à l'automne 1992, les hommes politiques hongrois étaient confrontés à une tâche politiquement risquée : le déficit budgétaire devait être réduit conformément aux normes approuvées par les organisations financières internationales, de façon à maintenir la crédibilité du pays ainsi que l'image du gouvernement comme celle d'un réformateur déterminé ${ }^{6}$. 
Après son retour de Washington, à l'été 1992, le Ministre des Finances annonça qu'il répondrait "non" à toute demande ou pression qui pourrait menacer dangereusement l'équilibre du budget pour l'année 1993. Un certain nombre de propositions impliquant des effets de redistribution importants furent avancées pour réduire le déficit budgétaire. Alors que la récession, les exonérations fiscales des investisseurs étrangers et les faillites massives, liées à une nouvelle loi sur les faillites, limitaient sensiblement les recettes du budget, il était évident que sans un ajustement radical des dépenses touchant le système de retraites, de santé, d'éducation et le système social - toute tentative de limitation du déficit serait vouée à l'échec. Quoi qu'il en soit, cette réforme budgetaire semblait comporter trop de risques pour un gouvernement confronté à des élections au début de l'année 1994. Dans ce contexte, le Ministère des Finances hongrois, encouragé par le FMI, élabora un programme budgétaire d'austérité centré sur les recettes et visant une réforme de la TVA. Aussi conformément à la nécessité d'harmoniser le système hongrois avec les normes de l'Union Européenne, la réforme introduisit un système à deux niveaux, unifiant les taux de TVA à $8 \%$ et à $25 \%$ qui remplaçaient ainsi les anciens taux ${ }^{7}$. Aussi, le plan de réformes incluait-il la suppression de toute exonération d'impôt, et l'augmentation de certains impôts autres que la TVA. En effet, les impôts sur la consommation d'alcool, de tabac et de pétrole devaient augmenter, et les taxes sur les voitures devaient être étendues à toutes les catégories de véhicules. De plus, les exonérations d'impôt destinées à favoriser la construction et la rénovation des maisons individuelles devaient être supprimées. Ces mesures d'accroissement des recettes, se combinaient à des coupes relativement faibles, mais sensibles, dans les dépenses : gel complet des dépenses de matériel, ainsi que des salaires dans les institutions publiques, à leur niveau de 1992; diminution substantielle du financement des programmes pour l'emploi de chômeurs ; ajustements d'urgence des coûts salariaux des Chemins de fer hongrois - impliquant le licenciement de 20000 personnes et la rationalisation de ses infrastructures sociales - ; enfin, gel des allocations familiales en fonction du nombre d'enfants recensés en 1992. Ce programme avait sans aucun doute des conséquences sévères sur la redistribution, et les responsables politiques avaient à répondre de cette question cruciale: qui devait supporter le coût de cette politique ? Le programme de réformes comportait donc, dès le début, des mesures compensatoires visant à devancer les contestations politiques contre l'inflation et les implications sociales du programme d'ajustement. Le plan prévoyait un choix entre l'augmentation de l'exemption d'impôt sur le revenu des ménages avec enfants, l'augmentation des allocations familiales selon le nombre d'enfants, calculées d'après les différents revenus des ménages, ou le maintien des incitations fiscales pour la construction et la rénovation des maisons individuelles, ou encore toute combinaison possible de ces mesures compensatoires, entre elles ou avec d'autres. Les mesures d'austérité et de réformes, et les mesures compensatoires devaient ainsi être inclues dans le projet de budget 1993.

6 Les différentes formes de protestation Malgré les compensations promises, une vague de protestations s'éleva après la présentation du programme d'austérité. En effet, la crainte de nouvelles pertes s'ajoutait à des doléances plus anciennes, et conduisirent à des formes variées de protestation : grèves de la faim dans la sphère civile, menaces de grèves et de manifestations dans le monde syndical, et menaces de vote contre les ajustements, y compris par les députés de la coalition. Les grèves de la faim Suite à l'échec des négociations avec le Ministère des Finances, le 22 octobre 1992, une grève de la faim de masse, coordonnée par une organisation civile, "L'association de 
personnes vivant en dessous du seuil de pauvreté" (LAÉT), débuta dans le village provincial de Bicske) . Les six grévistes exigeaient la suppression des réformes de la TVA et le maintien d'une TVA nulle sur les produits alimentaires de première nécessité, les médicaments et l'énergie domestique. Dès le début, la contestation avait gagné le soutien de certaines associations civiles, telles "l'alliance indépendante pour une représentation populaire", "la ligue pour la protection des intérêts des chômeurs", "la ligue pour la protection légale", "la chambre sociale" et "le parlement des gitans". Plus tardivement, plusieurs autres organisations rejoignirent le groupe des sympathisants, comme "les retraités de la Fédération nationale des syndicats hongrois" (MSZOSZ), l'organisation de Borsod-Abaúj-Zemplén de "la ligue démocratique de l'union des syndicats indépendants", la branche des ouvriers de l'acier de la MSZOSZ, "le parti social-démocrate hongrois", "l'association des citoyens libéraux - le parti des entrepreneurs", "l'association des contribuables hongrois", et "le parti socialiste hongrois". Les grèves de la faim se multiplièrent rapidement dans le pays. En effet, le nombre des personnes faisant la grève de la faim, ainsi que des personnes susceptibles de les remplacer en cas d'urgence atteignit rapidement des centaines, et au plus fort des protestations, des milliers. Le mouvement affecta un nombre toujours croissant de localités, dont la capitale ainsi que des petites et grandes villes de province. Plus le mouvement s'étendait, plus ses exigences se faisaient précises. Ainsi, les grévistes répertorièrent vingt produits alimentaires de base pour lesquels la TVA devait être de $0 \%$. En outre, ils exigeaient toujours plus de publicité dans les medias, et une couverture juste et sans manipulation du mouvement ${ }^{8}$. La contestation fit aussi plus de bruit. Prenant appui sur des manifestations de sympathie de certains syndicats de branche et d'autres syndicats locaux, les grévistes de la faim menacèrent le gouvernement de provoquer une émeute alimentaire, associée simultanément avec d'autres manifestations, et de provoquer des grèves dans des branches stratégiques de l'économie. Ils gagnèrent la sympathie avant tout de la presse écrite indépendante (et la presse de gauche), et leur leader, Ferenc Valencsik, devint rapidement connu, et même en quelque sorte respecté dans tout le pays. Une brève présentation des principales caractéristiques de la société civile hongroise peut aider à comprendre les organisations, les coordinateurs et tous ceux qui soutinrent les grévistes de la faim. Ainsi, la chute du régime communiste fut directement précédée et suivie par un développement impressionnant de la société civile, par la mise en place tant par le nombre que par les activités d'associations et autres organisations. De 1989 à 1992 le nombre d'organisations et de groupes d'intérêt connus dans les domaines tels que l'environnement, la santé, la jeunesse, le voisinage, les loisirs, les intérêts économiques, la religion, et dans une grande diversité d'autres domaines, a plus que doublé. On avait recensé 19950 associations en août 1992, contre seulement 8514 en $1989^{\circ}$. Cependant ce secteur civil avait assez mauvaise réputation, car la plupart de ses nouvelles organisations souffraient d'une légitimité et d'une représentativité douteuses. Il devait affronter la suspicion de l'administration, des partis politiques et des syndicats de travailleurs (ces derniers ayant à combattre des problèmes similaires dus à leur propre transition). D'après un haut fonctionnaire : "Le problème le plus difficile est peut-être d'identifier les partenaires du dialogue social. Ce changement radical a fait émerger quantité d'associations, et celles déjà existantes se sont elles aussi transformées. Les ministres ignorent l'existence de tout un ensemble d'organisations, alors que de nombreuses autres semblent manquer à la fois de membres et de soutiens. (Quoi qu'il en soit, une organisation composée d'un seul membre unique peut quelquefois avoir 
accès aux médias)" ${ }^{10}$. Le manque de légitimité et de soutien réel, caractéristiques de l'ensemble du secteur civil, était également le lot des nombreuses organisations participant au mouvement de contestation. A cet égard la LAÉT elle-même n'était pas totalement différente, bien que le déclenchement de la grève de la faim, sa coordination durant presque deux mois, et l'organisation de la publicité dans un monde de média en partie hostile, révélaient de réelles capacités d'organisation. La LAÉT avait été fondée à la fin du mois d'août 1991 et, selon l'un de ses leaders les plus connus, "rassemblait les membres déçus du MDF", dont un député, pour qui "le pouvoir manquait de sensibilité sociale". De cette façon, leur objectif restait l'action contre l'appauvrissement en Hongrie: la Croix Rouge hongroise fournissant un local. N'exigeant aucune cotisation de ses membres, tout en ne fournissant pas non plus de carte d'adhésion, la LAET revendiquait la possession d'un registre de ses membres, privilégiant la grève de la faim. C'est donc probablement un très faible nombre d'activistes qui est à l'origine de ses activités sporadiques. Ces derniers devaient principalement préparer et distribuer des tracts sur la pauvreté en Hongrie, et tentaient de recueillir des fonds dans le monde des affaires ${ }^{11}$. Rien, dans l'histoire de la LAÉT ne laissait présager des capacités à organiser des actions de protestation d'ampleur nationale. Les menaces de grève Parallèlement à l'extension du mouvement de grève de la faim, un grand nombre de menaces de grèves, témoignèrent du mécontentement des travailleurs à l'égard des mesures d'austérité. Dans les secteurs de l'éducation et de la santé, les syndicats faisaient planer des menaces par des actions de contestation coordonnées, comprenant la collecte de signatures, des manifestations et des grèves. Ils furent rejoints par les syndicats des travailleurs de l'acier et du rail, prêts à se mettre en grève si les mesures prévues étaient appliquées sans aucune modification. Se fondant sur une demi douzaine de rencontres syndicales de la fin du mois d'octobre, le Président de la MSZOSZ, Sándor Nagy, annonça que la base de la plus grande confédération ouvrière était prête à mener, si la menace ne suffisait pas, des actions de force contre la politique gouvernementale, pouvant prendre la forme d'arrêt général du travail d'un quart d'heure, de grèves longues, et de manifestations de rue massives $^{12}$. Là encore, une brève présentation des syndicats de travailleurs dans la Hongrie post-communiste peut être utile pour mieux comprendre l'origine et la signification de la menace de grève. L'actuelle structure du secteur syndical hongrois a émergé au cours l'été 1990. Alors que le taux de syndicalisation de la population active est passé de $90 \%$ dans les années 80 à $30 \%$ environ au début des années 90 , on estimait récemment à 1,5 million le nombre de syndiqués. Le mouvement syndical est devenu pluraliste ${ }^{13}$. Trois groupes syndicaux peuvent être distingués. La fédération nationale des syndicats hongrois (MSZOSZ) a hérité d'une petite partie seulement de la base de la Fédération communiste des travailleurs, (SZOT), mais a conservé son infrastructure, ses biens, et toute son équipe de dirigeants et de bureaucrates, avec à sa tête, Sándor Nagy, ex-membre du Comité Central du Parti communiste. La MSZOSZ s'est alliée au Parti socialiste hongrois, successeur du Parti communiste (MSZP). Un deuxième groupe rassemble les "satellites" du SZOT : le Rassemblement des syndicats des intellectuels ouvriers (ÉSZT), La Confédération des syndicats autonomes, et le Forum de réconciliation des syndicats (SZEF). La MSZOSZ et les "satellites" sont nettement majoritaires parmi la base ${ }^{14}$. Un troisième groupe est constitué de trois nouveaux syndicats issus du processus de transformation: la ligue démocratique des syndicats indépendants (LIGA), l'Alliance de solidarité des travailleurs, et le Conseil des travailleurs. Privés dès le début de la période de transition, de l'expérience de la 
MSZOSZ et de ses associés, en matière d'infrastructure et d'organisation, et confrontés à la réserve voire à l'hostilité manifestées par le gouvernement et la plupart des partis politiques à l'égard du syndicalisme, ces nouveaux syndicats restèrent lourdement handicapés. Les syndicats restèrent largement dominés par les fédérations postcommunistes. alors que le pluralisme était la caractéristique au niveau national, la plupart temps un seul syndicat étant présent au niveau des branches et sur les lieux de travail. L'impression générale est celle d'un mouvement ouvrier qui s'inscrirait dans un processus de déclin en général, caractérisé cependant, à la fois par des forces et des faiblesses asymétriques. Pour autant que l'on considère le nombre d'adhérents, les capacités de mobilisation, la présence, l'activité et l'influence dans les relations quotidiennes sur les lieux de travail, le mouvement dominé par la MSZOSZ semble être plutôt faible. La force des syndicats serait plus prégnante, au niveau macro, dans les différentes organisations et commissions tripartites, là où l'avantage d'un leadership expérimenté et de la bureaucratie peuvent être exploités au mieux. Les syndicats étaient également très présents sur la scène politique, par leurs rivalités internes, dans leur compétition avec les partis politiques et les organisations civiles pour le droit de représenter des groupes de non-travailleurs (le secteur populaire en général, et les retraités en particulier). Parfois même, les syndicats prouvaient leur force politique lors des menaces de grèves pour contrer des mesures économiques ainsi que des attaques dirigées contre eux. Les menaces syndicalistes en Hongrie sont de loin restées symboliques. Les grèves ont été sporadiques, organisées principalement à l'échelon local, peu d'appels à la grève émanant de structures de branche ou nationales, mêmes pour les grèves les moins récentes. Les quelques appels à la grève générale, dont la MSZOSZ fut l'initiateur exclusif, échouèrent régulièrement. Si l'on tient compte de tout ce qui précède, il apparaît alors clairement que malgré l'hypothèse de base de la "littérature ", selon laquelle la grève générale ne témoigne pas d'une force syndicale mais plutôt de sa faiblesse, c'est plutôt l'inverse qui s'est produit dans le contexte hongrois : le niveau extrêmement faible de l'activisme en matière de grève ne peut pas être interprété comme l'indicateur de la force syndicale. Nous pensons que le fait même que la Hongrie n'ait pas connu de grèves était plus ou moins indépendant de la volonté des syndicats. Tel est le contexte général dans lequel il faut évaluer les risques réels de grèves engendrés par les mesures d'austérité de 1992.

7 Les menaces de vote parlementaire contre le plan d'austérité Les partis politiques ne semblaient pas, eux non plus, favorables aux mesures d'austérité. Ceci était bien compréhensible, pour les partis de l'opposition parlementaire, aussi longtemps qu'ils se sentaient concernés. C'est sans surprise qu'ils manifestaient leurs désaccords avec les détails du plan d'ajustement. Les réflexions des partis de la coalition gouvernementale étaient encore plus négatives que celles de l'opposition. Le Parti des petits propriétaires, fragmenté et populiste, et les Démocrates chrétiens, soucieux de donner l'image d'un parti sensible aux problèmes sociaux des classes populaires, de même que la faction pro-Csurka du MDF, annoncèrent leur rejet du programme d'austérité et du projet de budget 93, s'ils restaient inchangés. Le conflit social, et les tentatives pour le résoudre on peut aisément conclure de ce qui précède qu'une stratégie gouvernementale visant à imposer par la force un programme d'austérité était voué à l'échec. Dès lors, pour calmer les tensions politiques, le gouvernement adopta d'abord une stratégie de négociations au cas par cas. Les visites, les requêtes et les lettres des parlementaires tentaient d'adoucir le mécontentement des grévistes de la faim. D'ailleurs, vers la mi-novembre, le gouvernement se montra disposé à répondre aux 
demandes du mouvement, et à maintenir des exonérations de taxes pour 4 ou 5 produits alimentaires de base, à condition que la grève de la faim cesse. Aux tentatives du gouvernement pour mettre fin à l'extension de cette profonde contestation s'ajouta une réelle compétition au sein des partis et des syndicats : faire plus et mieux dans l'intérêt des pauvres. Le Ministre des Finances, Kupa, rendit lui-même de longues visites aux dirigeants des partis de la coalition pour marchander avec les partis et les factions de possibles modifications des mesures en échange de leur vote de soutien pour le budget. Les négociations continues avec le plus médiatique des syndicats, démontrèrent également la volonté du gouvernement de poursuivre la mise en oeuvre du programme dans un cadre pacifique. La tactique des négociations au cas par cas sembla en effet donner des résultats. La LAÉT répondit aux promesses gouvernementales d'intégrer leurs revendications dans le programme d'austérité et de compensation, en faisant connaître sa volonté d'arrêter la grève de la faim, et de trouver une solution au conflit. De plus, certaines menaces de grèves s'arrêtèrent vers la mi-novembre. Le 18 novembre 1992, il était clair, par exemple, qu'aucune grève ne se déclencherait chez les mineurs. Un accord entre le gouvernement et leur syndicat fut conclu, sur l'un des points les plus conflictuels de la politique, et sur la poursuite des négociations jusqu'au début de décembre ${ }^{15}$. Un accord similaire sur l'ajournement de la contestation fut conclu dans la branche fer-acier, pendant que les employés de la santé et de l'éducation semblaient attendre impatiemment d'autres accords répondant à leurs revendications. Pourtant, les négociations échouèrent avec les employés du Chemin de fer. Ainsi, à l'époque, il était peu probable aux yeux des acteurs, que le Ministre des Finances, parvienne à convaincre les factions de la coalition de ne pas s'opposer à l'inévitable programme d'austérité. En dépit du succès partiel du marchandage au cas par cas, le baromêtre politique continuait à annoncer des avenirs tourmentés, et les tensions faisaient planer la menace de la formation d'une coalition anti-austérité par certains opposants ${ }^{16}$.

Les négociations tripartites des 20 et 21 novembre Les intérêts des syndicats ouvriers Dans cette atmosphère lourde de tensions sociopolitiques, l'appel des syndicats, au mois de novembre, pour résoudre tous les problèmes par des négociations tripartites et la signature d'un pacte social, semblait tomber à point pour le gouvernement - et pour les syndicats eux-mêmes d'ailleurs. Après de brèves discussions, un accord fut conclu rapidement pour conduire les négociations, dans le cadre déjà existant du Conseil pour la Réconciliation des Intérêts (CRI) et une date fut arrêtée les 20 et 21 novembre 1992. Il n'est pas étonnant de voir les fédérations de travailleurs négocier, plutôt que de poursuivre les conflits à leurs limites extrêmes. Il était évident pour tout le mouvement, et plus particulièrement pour la MSZOSZ, qu'ils auraient plus à gagner par la négociation et le compromis, qu'en prolongeant des grèves aux issues incertaines. La modération des syndicats a sûrement payé au niveau politique, à ce moment là. Dans une crise économique grave, et face au caractère exclusif de la politique gouvernementale, les syndicats ont toujours dû se battre contre leurs sérieux handicaps de légitimité et d'organisation, profondément enracinés dans leur état de transition. Les syndicats avaient aussi en vue de sérieux gains institutionnels. En choisissant le CRI comme plateforme des négociations, le forum tripartite pouvait apparaître publiquement comme un corps unique et solide, par lequel d'importants ajustements politiques pouvaient être menés. Quant aux membres du CRI, ils pouvaient donner l'image de pouvoir contrôler de façon non négligeable les décisions en matière de politique économique. Une autre conséquence portait sur la décision simultanée des 
intérêts directement représentés et non représentés lors des négociations. La façon dont les partenaires de la négociation ont été sélectionnés, et d'autres exlus, eut une grande importance, en particulier pour le CRI réellement disposé à jouer un rôle dans le choix des groupes sociaux auxquels seraient attribués des compensations aux pertes provoquées par l'austérité économique, et dans la forme même de cette compensation. Choisir le CRI comme forum pour le compromis social sur l'austérité a donc une signification qu'on ne peut comprendre sans un bref extrait de son histoire institutionnelle. Le statut du CRI Le prédécesseur du CRI, le "Conseil National pour la Réconciliation des Intérêts", avait été fondé en Octobre 1988. Son objet était de fournir un forum pour permettre les accords sur l'augmentation des salaires et les exemptions à la réglementation salariale. Cependant, le NCRI dut bientôt aborder d'autres sujets, comme les prix, les impôts sur les profits et les impôts sur le revenu... La négociation nationale sur les salaires, dont les consultations générales portèrent sur les domaines de l'économie et du social (voire même sur la définition commune des thèmes économiques et sociaux) resta un des problèmes principaux pour le CRI ${ }^{17}$. Après 1990, le CRI se soumit à un processus généralisé d'institutionnalisation, qui indiquait: 1) l'établissement des règles, des limites et du cadre de son contrôle sur la définition de la politique économique gouvernementale. En matière d'organisation, plusieurs comités tripartites furent mis en place au sein du CRI, ayant pour objet: la consultation de la politique économique, la politique salariale, les relations avec le monde du travail, les relations avec le marché du travail, les politiques sociales, l'information, l'éducation, la formation, et les privatisations. 2). l'institutionnalisation du statu quo tripartite, c'està-dire l'établissement de la liste définitive des organismes autorisés à prendre part aux activités du CRI. Ceci impliquait la participation de la MSZOSZ, la MOSZ, l'ÉSZT, les AUTONOMES, la LIGA, SOLIDARITE et le SZEF du côté des syndicats, tandis que les participants du côté des affaires étaient la ÁFEOSZ (la féderation nationale des coopératives générales de consommation), la IPOSZ (la Féderation nationale des associations hongroises de l'industrie - La chambre hongroise des artisans), la KISOSZ (l'organisation nationale de petits commerçants), la MAOSZ (La Féderation nationale des travailleurs), la MGYOSZ (Alliance nationale des industriels hongrois), la MOSZ (l'alliance nationale des producteurs agricoles et des coopératives), la féderation nationale de l'industrie, et la VOSZ (la féderation nationale des entrepreneurs). Le gouvernement assurait sa représentation par le ministre responsable des questions négociées au CRI. Alors que la MSZOSZ était l'organisation la plus puissante du côté syndical, son adversaire dominant dans le camp des affaires était la MAOSZ, qui regroupait le plus d'entreprises publiques, semi-privées ou privées en partie seulement. Pour évaluer correctement la structure et les interlocuteurs qui prenaient part à la table des négociations au CRI, il faut souligner que $80 \%$ des membres de la MSZOSZ étaient employés dans les entreprises représentées par la MAOSZ dans le CRI. D'après les observateurs, la présence de la MAOSZ au sein du CRI s'assimile plus facilement à celle d'un groupe de pression, qu'à celle d'un représentant du patronat face aux intérêts des employés ${ }^{18}$. Un des traits de comportement type du côté patronat est sa passivité tout au long des négociations tripartites de novembre 1992. Les intérêts du gouvernement A l'opposé des syndicats, les motivations réelles qui ont poussé le gouvernement à négocier semblent à première vue moins évidentes. Une zone d'ombre subsiste, même si les plus hauts dignitaires du gouvernement, comme le ministre des Finances, insistèrent sur la nécessité de maintenir la paix sociale. L'exécutif s'est donc bien senti contraint de négocier, sous la pression des menaces évoquées ci-dessus. On 
peut cependant douter d'une réelle crainte du gouvernement Antall, de voir émerger de graves conflits sociaux. Tout semblait indiquer que la LAÉT, organisatrice des protestations les plus radicales, devait être présente à la table des négociations. Cependant, choisir le CRI comme forum des négociations, revenait automatiquement à décider qu'aucune organisation représentant la société civile, à l'exception des associations économiques et des syndicats, ne participerait à la solution du conflit. Malgré les réclamations répétées, les menaces et les plaintes de la LAÉT, l'organisation locomotive de la grève de la faim n'a réussi à obtenir, ne serait-ce qu'un statut d'observateur, lors des négociations sur le pacte social. Le gouvernement devait penser que les grèves elles-mêmes poseraient plus de difficultés au programme d'austérité et à la paix sociale que les grèves de la faim. Cette explication est malgré tout insuffisante. Premièrement, le gouvernement doutait pertinemment des capacités réelles des syndicats à paralyser l'économie. Il aurait été suffisant, en l'absence d'autres sources, de se fier à la presse pour deviner les difficultés que rencontraient les syndicats pour s'accorder sur la nécessité des grèves. Le leader de la LIGA de la mine de charbon de Balinka déclarait: "Nous voulons agir au niveau des négociations et des accords. Je crois qu'un appel à la grève aujourd'hui ne pourrait être pris au sérieux, car il serait facile pour le pays d'importer du charbon moins cher d'Ukraine ou de Slovaquie" ${ }^{19}$. Deuxièmement, comme cela a été évoqué plus haut, à partir du moment où les pourparlers du pacte social débutèrent, certaines menaces de grèves furent aussitôt neutralisées. Troisièmement, si le gouvernement souhaitait réellement voir aboutir un tel pacte, pour mettre fin à toutes les menaces de grève, il dut vite apprendre, malheureusement pour lui, que l'aménagement tripartite ne séduisait pas tous les syndicats de branche. Ainsi, dès le premier jour des négociations, les syndicats des chemins de fer annoncèrent une grève préventive de deux heures. Apprenant cela, le Ministre des Finances Kupa, chef de la délégation gouvernementale, posa cette question pertinente: "Pourquoi devrions nous aboutir à un accord, si la grève continue au niveau des branches, et s'il n'y a pas de paix sociale ?" ${ }^{20}$. Pourtant, le ministre se calma bien vite et poursuivit avec obstination cet étrange marchandage, qui ne pouvait empêcher ni les grèves dont l'annulation était une condition pour le pacte, ni celles qui se déclenchèrent dès la signature du document. Nous pensons que, dès le début du mois d'octobre 92, l'enjeu n'était pas simplement et directement de garantir la "paix sociale", mais plutôt l'équilibre global du pouvoir entre le gouvernement, la coalition et le Parlement. Le gouvernement Antall et plus particulièrement les technocrates réformistes craignaient moins la contestation civile et syndicale que l'effritement de la coalition, lié directement au sort du programme d'austérité, du budget 93, et plus globalement lié au sort du gouvernement lui-même. L'intérêt véritable du gouvernement et de la technocratie était très certainement de se servir du pacte comme de la preuve d'une acceptation sociale de leurs mesures, afin de convaincre et maitriser la fragile coalition. Tel est le contexte qui permet de comprendre plus facilement comment et pourquoi les menaces symboliques des syndicats et leur poids ont été surestimés, les gratifiant dès lors d'une position de force durant les négociations. Les gagnants et les perdants du pacte Les mesures d'austérité et de compensation approuvées par le pacte social du 21 novembre diffèrent significativement des plans initiaux du gouvernement. La nouvelle structure approuvée des gains et des pertes, comprenait des enjeux socio-politiques complexes, reflétant par là même, à la fois l'influence relative des différents groupes dans l'arène politicoéconomique hongroise, et également le sens de leurs querelles politiques dans ce 
contexte. En toute légitimité, les syndicats ouvriers crièrent victoire. Ils purent obtenir beaucoup, en termes de compensation financière pour leur base, et en termes de récompense politique pour les organisations elles-mêmes. Pour illustrer ces derniers gains, citons : la suppression du contrôle des salaires, élément hétérodoxe et central dans la politique de stabilisation du gouvernement Antall ; l'augmentation substantielle du salaire minimum ; le maintien de certains encouragements fiscaux à la construction et la rénovation de maisons individuelles; la réintégration d'un impôt préférentiel pour les cols blancs; l'augmentation des allocations familiales et des préférences fiscales liées au nombre d'enfants; la promesse de salaires plus élevés dans les domaines de la santé et de l'éducation; la baisse du taux de TVA le plus bas, des $8 \%$ envisagés à $6 \%$; et le maintien des taux nuls pour les médicaments et l'énergie domestique. Le retrait du projet de loi très interventionniste, sur les élections syndicales et la division de la propriété syndicale, put être considéré comme le prix politique à payer lors des négociations. D'après certaines analyses, ce retrait fut le résultat le plus important pour les syndicats ${ }^{21}$. En effet, l'un des syndicats, Solidarite, qui avait, la mort dans l'âme, quitté le CRI avant la fin des négociations, accusa violemment ses congénères de sacrifier l'économie à la politique ${ }^{22}$. (Solidarite fut radiée peu après du CRI, pour résistance et incapacité à coopérer, et parce que ses objectifs et ses méthodes étaient inacceptables pour les six autres syndicats. Prenant des positions politiques de plus en plus extrémistes, Solidarite perdit ses caractéristiques syndicales, et en 1994, la presse l'accusa de coopérer avec le parti pro-faciste hongrois). Le gouvernement, et plus spécialement les réformistes dirigés par Kupa, pouvaient eux aussi tranquillement célébrer leur victoire. En premier lieu, ils tirèrent bénéfice de l'acceptation d'une réforme fiscale modifiée, et d'autres difficiles mesures syndicales, dont l'augmentation substancielle de la contribution des entreprises et des travailleurs en faveur du fond d'aide sociale pour les chômeurs. En second lieu, le mécontentement de la coalition vis à vis des mesures d'austérité et du budget avait été atténué par le pacte, et le gouvernement pouvait compter sur le soutien au Parlement pour le vote du budget 93. Enfin, Kupa et le gouvernement purent faire la preuve de la force du pouvoir gouvernemental, ou du moins de sa capacité à résoudre une crise sociopolitique. Ils ont par là même amélioré leur image d'hommes politiques prenant les partenaires sociaux au sérieux, et de négociateurs confirmés, sans imposer de politique de manière coercitive. Ce n'est pas une surprise si les gagnants ont accueilli le pacte avec un enthousiasme mitigé, alors que les perdants, les grévistes de la faim, les organisations civiles et les groupes populaires qui les soutiennent, observaient, à juste titre, que le pacte social avait été biaisé, au profit des classes sociales aux revenus moyens, qui avaient plus de poids politique, et étaient mieux représentées lors des négociations. D'une part, la seule revendication caractéristique spécifiquement associée au mouvement des grèves de la faim - à savoir un taux de TVA nul pour les produits alimentaires de base - s'est trouvé quelque peu noyée dans le labyrinthe des négociations tripartites. C'est un taux de 6\% qui leur sera appliqué. D'autre part, la plupart des accords conclus n'étaient guère tournés vers un souci d'aide aux plus pauvres ${ }^{23}$. De plus, dans le processus de préparation du pacte social, les organisations civiles devaient faire face non seulement à une critique de leurs méthodes par le plus fort des syndicats, mais également à leurs innombrables efforts pour monopoliser une population, les retraités en particulier. Bien évidemment, ils se sentirent trahis vers la fin de ce processus préparatoire, et continuèrent à protester mais par des moyens de plus en plus "désespérés". L'après-pacte : la tentative pour un référendum Déçue par 
son exclusion du pacte social, la LAÉT prit l'initiative d'organiser une nouvelle action de contestation, afin de montrer son désaccord avec la politique économique du gouvernement et la forme du système institutionnel démocratique hongrois en général. Aidée par de nombreuses autres organisations civiles, ses activités commencèrent le 15 décembre 1992, par le recueil de signatures pour l'organisation d'un référendum sur la dissolution du Parlement et en faveur de nouvelles élections générales: "Dans sa structure récente, le Parlement a prouvé son incapacité à résoudre les problèmes de près de quatre millions et demi de Hongrois vivant en deçà ou au niveau du seuil de pauvreté" 24 . Cette initiative connut très vite un succès considérable. Comme indiqué dans le tableau ci dessous, trois semaines suffirent à la LAÉT et ses associés pour recueillir les 100.000 signatures nécessaires à valider le référendum. La popularité du projet atteignit non seulement les groupes sociaux appauvris, mais également tous les hongrois déçus par le processus de transition en général ${ }^{25}$.

Date Nombre de signatures de citoyens collectées 15 décembre 402129 décembre 81 7005 janvier 958007 janvier 10320019 janvier 172000

La situation politique fut encore plus tendue quand cette action de la LAÉT fut rejointe par des exigences et des menaces émanant $d$ 'autres organisations. Lors de son congrès du 5 décembre 1992, l'Alliance nationale des prisonniers politiques (POFOSZ), alors très médiatique, proposa au Parlement d'amender les lois électorales sur trois points fondamentaux : la création d'une deuxième Chambre pour les organisations en dehors du système formel des partis, la réintroduction de la régle sur la révocation des députés, et l'élimination des listes de partis au sein de la procédure électorale. Le congrès, en plébiscitant István Csurka avec un enthousiasme débordant, menaça le Parlement de recueillir des signatures s'il ne prenait pas en considération leurs propositions ${ }^{26}$. L'initiative du POFOSZ renforça l'image générale de succès des actions de la LAÉT. Le cadre politique et institutionnel de la démocratie hongroise n'ayant encore été ni légitimé ni consolidé, il fut contesté et défié par de larges groupes sociaux au sujet de ses principes fondamentaux ${ }^{27}$. Le succès inespéré de l'initiative prise par la LAÉT provoqua des réactions mitigées de la part des partis établis et plus généralement de l'élite intellectuelle et politique hongroise, suscitant en même temps mauvaise conscience, incertitute et aversion pour le référendum. La mauvaise conscience provenait peut-être de l'échec de l'élite pour définir les moyens et les voies institutionnelles pour l'exercice du contrôle civil de la politique de l'Etat dans la constitution. Cela conduisit à la multiplications de débats médiatisés sur les origines, les fins et les résolutions de la crise. L'origine du sentiment d'incertitude vint du statut légal très contesté, du référendum. Si ce dernier était, de fait, légal, le Comité Constitutionnel du Parlement n'arrivait pas à se prononcer sur sa conformité par rapport à la constitution. Pour en décider, ce dernier pria le Conseil Constitutionnel de juger de la constitutionnalité du référendum. L'aversion pour le référendum fut un sentiment partagé par la plupart des partis de la coalition et de l'opposition. Ils exprimèrent à plusieurs reprises leur désaccord en soulignant les risques liés à cette initiative: l'anarchie et le chaos politique, la nature anticonstitutionnelle du référendum, et les menaces de fragilisation du système démocratique institutionnel en général. Sans aller jusqu'à évaluer la validité de ces craintes, il suffit ici de souligner la volonté manifestée par le système politique d'opter pour le statu quo, et contre les revendications populaires pour un nouveau modèle de système politique ${ }^{28}$. (En plus de leur engagement pour préserver les régles du jeu démocratique existantes, la plupart des partis se sentaient insuffisamment préparés aux élections de 1993). Le désintérêt et 
la réserve des partis ont certainement eu des conséquences importantes sur l'aboutissement de cette initiative. A la mi-janvier 1993, alors que l'idée du référendum était soutenue par 172.000 citoyens, la Cour Constitutionnelle décida qu'il n'était pas constitutionnel. Alors même que le réferendum était légal, c'est le projet de loi en tant que tel qui était supposé violer la Constitution. La Cour exigeait du Parlement qu'il amende ce projet de loi avant la fin 1993. Les partis représentés au Parlement et d'autres acteurs du système politique établi accueillèrent cette décision avec beaucoup de satisfaction. La collecte de signatures perdit de son élan, et cessa rapidement. Nombre d'organisations civiles qui avaient soutenu ce gigantesque mouvement, disparurent. La LAÉT elle-même s'engagea à fonder un parti politique, dont on n'entendit pas parler lors des élections de 1994.

11 Enseignements à tirer du cas hongrois Tirer les enseignements du cas présenté ici nécessite de réfléchir sur certains concepts et postulats utilisés dans les travaux exitant sur les pactes sopciaux, sur les politiques "négociées", "consultées" ou "interactives", et sur les types de démocratie. "Force gouvernementale" - "force syndicale" Les analyses sur les compromis néocorporatistes, qui comprennent également les pactes sociaux, insistent souvent, d'une part, sur la capacité et l'engagement du gouvernement, et d'autre part sur la centralisation et la concentration organisationnelle ainsi que sur le monopole de la représentation des syndicats et du monde des affaires, comme conditions préalables à une concertation réussie. Capacité et engagement du gouvernement sont alors synonymes de la volonté et de l'aptitude de ce dernier à initier, élaborer et rendre effectives les politiques économiques, à contrôler et faire valoir à son avantage les accords à négocier ${ }^{29}$. A son tour, la centralisation concertation et le monopole de représentation syndicale empêchent les accords conclus au niveau macro-économique d'être remis en cause par les leaders de second rang, le commun des troupes ou d'autres organisations rivales ${ }^{30}$. D'autres analystes emploient plutôt les termes de force gouvernementale ou syndicale (ou du monde des affaires) pour évoquer les qualités ci-dessus. Dans cette formulation plus simple ce sont les gouvernements forts (au niveau politique et organisationnel) et les syndicats forts (au niveau politique et organisationnel) qui ont le plus de chances de faire aboutir de façon positive les concertations et de signer des conventions sociales ${ }^{31}$. Les concepts évoqués plus haut ont en commun leur tendance à assimiler les notions de "capacité" ou de "force" aux conditions requises par chaque négociateur pour garantir le succès de la concertation. Il en découle une définition "absolutiste" des qualités nécessaires aux négociateurs respectifs. Or, avec ou sans concertation, gouvernements et syndicats peuvent être crédités d'être "forts" ou "faibles", d'être "capables" ou "incapables" de mettre en forme, de rendre effectives et de contrôler les politiques. Ils peuvent être "centralisés", "monopolistes" ou "décentralisés" et «fragmentés ». Seulement, si l'on ne tient pas compte d'éléments contextuels plus stricts, on peut se demander quelle pertinence ont ces notions de force et de capacité, en particulier s'il s'agit d'une concertation sociale réussie. Cette remarque est d'autant plus nécessaire que la concertation et les conventions sont des activités de coopération, exigeant une multitude d'échanges entre les partenaires, ainsi qu'une évaluation comparative, mutuelle et permanente des forces et faiblesses de chacun ${ }^{32}$. De plus, la concertation et la convention mêmes peuvent modifier - accroître ou diminuer - la force et la capacité des partenaires. Le cas hongrois met alors l'accent sur la nécessité de s'arrêter sur certains éléments de la définition de la force et de la capacité, et aussi sur les 
perceptions mutuelles concernant cette capacité, comme conditions pour des pactes réussis.

Si l'on s'en tient à la définition « absolutiste » telle qu'elle était donnée ci-dessus, ni le gouvernement ni les syndicats en Hongrie n'auraient pu être définis à aucun moment comme capables ou forts. Ceci est d'autant plus vrai concernant la "force" de l'aile réformiste et technocratique du gouvernement Antall. Ils ont pourtant su négocier et signer un pacte, parvenir à une résolution pacifique d'un conflit sociopolitique, et se prémunir d'initiatives réformistes dans quelques domaines ${ }^{33}$. On sait aussi pertinemment que le gouvernement et les syndicats ont exhibé un certain type de capacité ou de force, ce qui a largement facilité leur tâche. La force des technocrates financiers du gouvernement était certainement relative : on ne pourrait l'évaluer qu'en termes comparatifs. En effet, d'un point de vue politique et organisationnel, le groupe des réformistes avait des faiblesses, mais également des points forts, doté d'une plus grande capacité d'analyse et de perspicacité, et d'une cohérence bureaucratique, caractéristiques qui faisaient défaut à ses adversaires: les groupes rivaux de la bureaucratie, les organisations civiles de soutien aux actions de protestation, les factions extrémistes et déçues de la coalition, et les partis de l'opposition. Ces remarques peuvent tout aussi bien s'appliquer aux syndicats. Si ceux-ci étaient suffisamment faibles (particulièrement la MSZOSZ), ils étaient pourtant beaucoup plus forts que leurs plus fervents rivaux de la société civile, ou à la marge du mouvement syndical. C'est leur force relative, d'un point de vue organisationnel et politique, qui les constitué en partenaires valables pour une solution au conflit.

13 Les forces respectives du gouvernement et des syndicats devraient être (et ont été) , elles aussi, évaluées en fonction de leurs capacités réciproques. C'est la comparaison entre les perceptions qu'avaient les partenaires de leur propre force et de celle des autres, qui constitue un élément important. Si en 1992 la force (politique et organisationnelle) du gouvernement Antall, comparée à celle des syndicats, avait été plus importante, le gouvernement aurait pu se passer de négociations, mais il essaya d'imposer sa politique par étape et par la manière forte, en pensant recourir à des mesures coercitives en cas de protestations syndicales ${ }^{34}$. Réciproquement, si les syndicats avaient été beaucoup plus forts que le gouvernement, on aurait alors assisté, peut-être, au scénario polonais de 1989 : les syndicats auraient évincé le gouvernement, plutôt que - ou après avoir - engager des négociations. Mais ce qui s'est réellemnt passé en fait, c'est qu'un gouvernement faible (mais relativement habile) et un mouvement syndical faible (mais assez organisé) se sont mutuellement confortés et légitimés au sein même de leur contexte respectif de structure de pouvoir politique et organisationnel. (Et en définitive, ce sont les deux acteurs les plus importants du pacte qui en profitèrent le plus, à savoir la technocratie financière du gouvernement, et la MSZOSZ). Ou pour le dire autrement: le gouvernement utilisa cet accord avec les syndicats comme un bouclier pour préserver la politique économique des pressions conjointes des partis politiques et des organisations civiles radicales. Les syndicats utilisèrent, à leur tour, le pacte avec le gouvernement comme prétexte à leurs efforts pour monopoliser la base, pour résoudre les conflits sur la scène syndicale, et pour évincer les organisations civiles rivales.

14 Le style politique et le développement de la société civile Selon le point de vue le plus répandu, fondé sur l'expérience du Tiers Monde, mais adapté au contexte de l'Europe de l'Est, il existe une relation étroite entre la manière de conduire une politique 
économique et la recherche d'une démocratie viable. Le postulat est le suivant: le caractère "insulaire" et "impératif" des décisions de choc prises dans le domaine économique dans les pays post-communistes, peut nuire au développement de la démocratie, car celles-ci empêchent les organisations civiles d'émerger dans un processus interactif avec les responsables politiques. Le caractère péremptoire d'une politique est réputé incompatible avec le développement "d'acteurs stratégiques". Par contre, les politiques "consultatives" et "négociées" sont censées faciliter le processus démocratique, car elles créent des "acteurs stratégiques", en encourageant les organisations civiles à développer, articuler et formuler les revendications politiques, et en aidant la société à participer aux étapes de la transformation économique, de l'intérêt commun ${ }^{35}$. Si l'on s'attache au cas présenté plus haut, on peut, semble-t-il, poser un regard critique sur le postulat dont il est question ici. La différenciation simpliste entre d'un côté les conséquences de la conduite "péremptoire" de la politique économique sur le développement de la société civile et la démocratisation, et de l'autre les conséquences de la méthode "consultative" pour mener cette politique, ne tient pas. Chaque style politique peut préselectionner certaines organisations civiles (et civiques) qu'il voudrait voir émerger, et d'autres qu'il voudrait voir s'écrouler. Cependant, dans des contextes différents, des styles de politique peuvent soutenir ou contenir le développement de la société civile, et faire de cette optique la base d'une institutionnalisation démocratique. D'un côté, les stratégies d'ajustement néo-libéral, plus ou moins liées à des préoccupations humanitaires de redéfinition du soutien aux plus pauvres, peuvent, à l'évidence, favoriser l'émergence d'un secteur organisé, efficient et compétent d'ONG et d'ODR, spécialisées dans l'allocation de fonds sociaux, au niveau local notamment. Ceci peut avoir des effets positifs au regard des besoins économiques et du développement politique ${ }^{36}$. Il n'y a guère de doute que ces politiques néolibérales portent souvent préjudice à d'autres organisations civiles, et plus particulièrement aux syndicats, en limitant généralement leur influence sur des décisions redistributives ${ }^{37}$. Mais d'un autre côté - et comme on a pu le voir dans le cas hongrois - cela n'est pas entièrement différent dans le style des décisions économiques plutôt "interactives" et "néocorporatistes". En sélectionnant les syndicats et les acteurs économiques - les partenaires sociaux traditionnels - comme partenaires des négociations, les politiques consultatives font appel à une fraction seulement de la société civile organisée, excluant le reste, à savoir les nombreux groupes sociaux disposant d'une représentation politique mineure.

15 Cette étude apporte finalement un soutien empirique à l'énoncé ci-dessus. Premièrement, j'ai montré comment, malgré de dramatiques contestations organisées, certaines revendications ont été négligées, et comment certaines organisations civiles ont été maintenues à l'écart du débat sur la politique économique, y compris quand le gouvernement Antall, réputé en général pour sa capacité à imposer sa politique économique fit un pas vers la consultation à l'automne 1992. Deuxièmement, le gouvernement n'était pas le seul à maintenir éloignées de la table des négociations les organisations civiles et leurs revendications spécifiques. Mobilisés par leur lutte pour la légitimité, leur exclusivité et leurs tentatives pour monopoliser leur base, et ceci durant toute la période de transition, les syndicats hongrois acceptèrent mal de partager le contrôle de la politique économique avec leurs rivaux, les représentants des intérêts de la société. Ils essayèrent donc de les dépasser en nombre et de laisser à l'écart leurs revendications. Troisièmement, on a pu observer, dans un contexte strictement politique, un type de comportement similaire, à la fois défensif et exclusif. 
Tout comme les syndicats, les partis politiques hongrois prouvèrent leur engagement à défendre un statu quo fragile, dès lors que les organisations civiles, frustrées par leur exclusion du tripartisme, continuèrent à protester en tentant d'obtenir l'organisation d'un référendum contre le système politico-institutionnel en place. Quatrièmement, les résultats peuvent apporter un éclairage sur l'importance de l'analyse des mécanismes politiques et institutionnels de la transition, à travers lesquels les différentes façons de mener une politique économique se répercutent sur la construction de la société civile en particulier, et sur la démocratisation ainsi que les relations Etat-société, en général. Le conflit et sa solution négociée doivent néanmoins être interprétés suivant un autre point de vue.

16 L'apprentissage par tatônnement de la démocratie Le processus présenté plus haut a-til contribué à renforcer ou à affaiblir les chances d'une consolidation et d'une institutionnalisation démocratiques en Hongrie? A cette question, la réponse tenderait à être plus positive que négative. Il est évident que le système démocratique hongrois est, aujourd'hui, loin d'être complètement institutionnalisé et consolidé. Mais il est est clair également que de novembre 1992 à janvier 1993, des étapes importantes ont très certainement été franchies dans cette direction, et que les menaces dangereuses qui planaient sur le processus de consolidation ont été neutralisées. Le premier résultat auquel est parvenu le processus de résolution du conflit tient en une avancée substancielle dans la consolidation du CRI en tant que forum institutionnel majeur, dans la reconstruction des relations gouvernement - acteurs sociaux - acteurs économiques; dans les discussions et la négociation des réformes politiques aux douloureux effets de redistribution; et si nécessaire, dans la résolution des conflits politiques d'envergure nationale. Cet aboutissement correspond au modèle aujourd'hui traditionnel qui sous-tend le développement démocratique en Hongrie : "la démocratie par des moyens non démocratiques"38. L'effet politique d'alors a été un équilibre temporaire des pouvoirs entre les organisations d'intérêt, l'exécutif et le législatif. Cet équilibre peut à nouveau être discuté et redéfini, en cas de nouveau développement disproportionné de la sphère politique et économique, modifiant la force de négociation de l'un ou l'autre partenaire. Deuxièmement, la société civile a dû apprendre deux choses pendant cette période: d'une part que la démocratie est un équilibre entre l'inclusion et l'exclusion, et d'autre part que ce qui différenciait les exclus des autres était la relativité du pouvoir politique, l'organisation, le contexte politique et institutionnel concret, et la situation économique générale. Avec une certaine amertume, la société civile devait aussi apprendre la signification du rythme chronologique de la démocratie, et la puissance des intérêts liés au maintien du calendrier démocratique. Les citoyens devaient faire l'expérience de leur incapacité à sanctionner le gouvernement pour son prétendu manque de sensibilité sociale, au cas où ils le souhaiteraient. Plutôt que de répondre à un référendum immédiat, il leur fallut attendre les prochaines échéances électorales. Troisièmement, les élites politiques ont expérimenté l'importance de renforcer et d'affiner les règles de fonctionnement de la démocratie. Le défi référendaire de la LAÉT obligeait les politiciens hongrois à renouveler de façon drastique leur engagement pour le modèle institutionnel démocratique donné, qui ne prévoyait que peu de place pour les actions démocratiques directes. Après la décision de la Cour Constitutionnelle du 19 janvier 1993, il devint clair pour tout le monde qu'il ne serait désormais plus légal de tenter une dissolution du Parlement par des moyens de démocratie directe. Réciproquement, le conflit a renforcé la Cour Constitutionnelle dans son rôle spécifique d'arbitre en dernier ressort dans la 
consolidation des institutions démocratiques en Hongrie. De toute évidence, les processus d'apprentissage sont très proches de la consolidation démocratique, et ils aident probablement plus rapidement qu'on pouvait l'espérer à sortir la société de son déséquilibre de "non-système" et de son état "fluide" liés au changement de système ${ }^{39}$. Si l'on accepte les arguments ci-dessus sur la consolidation du système politique, on peut quand même se poser la question suivante: Quel est ce système supposé se consolider en Hongrie? Plus précisément on peut se demander si l'équilibre entre les exclus et les inclus ne s'est pas cimenté trop hâtivement et parcimonieusement, si la défense et le maintien du statu quo n'ont pas exclus arbitrairement de groupes sociaux importants, et si ce système sera un vecteur suffisant au déploiement des "éléments libéraux de la démocratie" ${ }^{40}$. Pour le dire autrement, on peut s'interroger sur le type de démocratie, pour autant qu'il est possible de le faire pour la période actuelle, et sur les fondements du cas qui nous intéresse.

Vers un régime démocratique dual? Inclusion ou exclusion - contrôle social sur les décisions économiques - participation directe ou par représentation aux institutions démocratiques et au processus de leur création - tels étaient les thèmes les plus chaudement débattus dans le monde politique et social, et autour desquels s'est développé le conflit sociopolitique hongrois de l'automne 1992. Sans aucun doute, la combinaison et le partage des moments d'inclusion et d'exclusion d'un régime démocratique sont des caractéristiques suffisamment importantes pour faire partie des éléments principaux constitutifs de sa définition. Dans une typologie récemment élaborée par Carlos $\mathrm{H}$. Acuna et William $\mathrm{C}$. Smith, les régimes démocratiques sont catégorisés essentiellement d'après leur dessein à savoir l'engagement et la capacité à inclure les acteurs sociaux. En effet, ils définissent un régime d'après la manière dont les gouvernements réagissent face aux tensions sociales qui découlent de l'amélioration des performances économiques. Ils décrivent trois scénarios permanents possibles une démocratie fragmentée et recourant à l'exclusion, avec une économie néolibérale ; une démocratie tournée vers le concept d'inclusion, avec des acteurs forts et un Etat interventionniste; et des régimes démocratiques duals. Plutôt que d'évaluer la pertinence de cette typologie pour apprécier le régime démocratique hongrois, mon propos cherchera à formuler quelques réflexions sur le troisième type : la démocratie duale ${ }^{41}$. Pour Acuna et Smith, le régime démocratique dual est un scénario où "les élites de l'Etat cherchent à s'allier avec une minorité stratégique de l'opposition afin d'exclure la majorité des acteurs sociaux en place, en désintégrant et en neutralisant leur capacité à mener des actions collectives. Dans ce scénario, la stabilité économique et politique est facilitée par une logique de pouvoir étatique duale (respect de l'organisation de la minorité alliée, et désarticulation du reste), et par une distribution inégale des ressources (les bénéfices sont étendus aux seuls secteurs alliés dans les secteurs économiques et dans celui du travail organisé). Le caractère particulièrement élitiste de cette logique de gouvernement définit la dualité de ces régimes démocratiques... Bien sûr, tous les régimes revêtent plus ou moins cette dualité, car, toute stratégie d'inclusion implique nécessairement l'exclusion. Le rapport important d'exclusion/inclusion, la dépendance de la stabilité des régimes à ce ratio, et la volonté de la plupart des acteurs politiques de le maintenir, sont les éléments propres à différencier les régimes démocratiques duals des autres" ${ }^{42}$. Cette interprétation semble convenir à la situation hongroise présentée dans cet article. Bien que l'évaluation précise des détails sur les conséquences réelles de l'accord en termes de redistribution (conséquences économiques et politiques) exige des recherches plus approfondies, le 
cas probant présenté semble nous conduire à la conclusion selon laquelle l'accord de 1992 n'incluait qu'une minorité stratégique, tout en excluant "la majorité des acteurs sociaux restant, par la désarticulation de leur capacité à mener des actions collectives" $^{43}$. Estimer l'importance relative de la majoritée exclue et de la minorité incluse dépend en partie du choix d'une interprétation concrète. Si l'on admet l'argument de la LAÉT selon lequel les millions de Hongrois, qu'il dit représenter, ont été volontairement oubliés par les négociateurs de l'accord, ce sont alors l'ensemble des pauvres et des semi-pauvres de la société qui ont été exclus du compromis et du marchandage socio-politique. S'il est juste d'admettre que la structure des compensations a été biaisée en faveur des classes sociales moyennes et des classes moyennes inférieures, alors l'importance relative des exclus est encore plus grande: elle concerne une grande partie du secteur populaire. Si l'on fonde l'évaluation sur le nombre de groupes sociaux directement présents à la table des négociations, il faut alors comparer le million et demi de syndiqués (et environ un million d'employeurs), avec la majorité du reste de la société. Finalement, si l'on reprend l'argument radical, selon lequel les intérêts politiques et institutionnels de l'élite syndicale, ont fortement dominé la volonté de celle-ci de représenter les revendications de la base au cours des négociations, alors on brosse les traits d'un compromis réellement élitiste par nature ${ }^{44}$. Si l'estimation extrême de Solidarité était juste, le pacte portait principalement sur la compensation de l'élite syndicale des pertes économiques de leur base par des bénéfices politiques. Sans autre intention de qualifier avec précision la proportion des exclus et des inclus, il suffirait ici de se référer aux éléments élitistes de l'accord, décrits précédemment, et ainsi de caractériser à la fois la structure organisationnelle de ses participants, leurs comportements face à leurs rivaux, et la grande proportion de ceux qui ont le sentiment d'avoir été mis à l'écart, quelqu'en soit la juste interprétation. En outre le pacte et ses suites témoignent suffisamment des capacités "désarticulées" et "neutralisées" du reste des acteurs sociaux à mener des actions collectives; du lien entre la stabilité du régime et un taux élevé d'exclusion, et de l'intérêt de la plupart des acteurs politiques à maintenir ce dernier élevé caracéristiques élaborées par Acuna et Smith dans le contexte du scénario de la démocratie duale. On pourrait en fait trouver d'autres similitudes quant à l'évolution d'une situation confuse vers un type de démocratie duale. Le gouvernement n'a pas toujours suivi des politiques d'inclusion, comme cela a été le cas lors du conflit social de l'automne 1992. En 1990 et 1991, il n'était pas réellement engagé vers le dialogue social, il tentait plutôt une complète désarticulation et neutralisation des capacités des partenaires sociaux à mener des actions collectives. Le leader de la MSZOSZ, Sándor Nagy a seulement été intégré dans le cadre de consultations personnelles avec le Premier Ministre, Jósef Antall, lors des menaces de grèves proférées par la plus grande fédération ${ }^{45}$. Dans ce contexte, le changement d'attitude du gouvernement à l'automne 92 peut être interprété, comme le suggéreraient Acuna et Smith, comme le chemin d'une démocratie fragmentée et d'exclusion, vers un régime démocratique dual, où "le gouvernement sera obligé d'accorder de petites concessions à des minorités stratégiques, dont le soutien apporte la marge nécessaire au succès de la négociation de problèmes de réforme spécifiques"46. Il y a cependant certains points sur lesquels l'interprétation autorisée par le type dual de démocratie doit être modifiée et adaptée au cas hongrois. Premièrement, les balbutiements du gouvernement hongrois vers une inclusion "démocratique duale" n'ont pas débuté quand la situation économique commença à s'améliorer, mais plutôt au plus profond de la crise économique. 
Deuxièmement, malgré les tensions sociales croissantes, on pouvait se demander quel degré de tensions sociales il fallait atteindre pour engendrer un changement d'attitude du gouvernement. En outre, on peut se demander quels ont été les éléments particuliers de ces tensions qui ont poussé le gouvernement vers une position politique davantage tournée vers l'inclusion. Comme on le sait, les "crises" et les "tensions" n'expliquent pas seules les réactions et les comportements de ceux qui en sont affectés, et déterminent encore moins l'issue de la situation. Ceci est particulièrement évident dans une perspective comparative: alors que certains gouvernements et régimes prennent des mesures préventives dès les signes précurseurs d'un mécontentement populaire ou social, d'autres tolèrent des niveaux de tensions politiques et sociales relativement élevés, sans intention visible de les calmer. D'après les travaux sur les politiques d'ajustement et de stabilisation économique dans les "LDC", c'est plus la perception de la crise économique, que la crise elle-même, qui peut influencer la programmation des étapes décisives du gouvernement, leur contenu et leur style ${ }^{47}$. Il ne doit pas en être autrement concernant les tensions sociales et les crises sociopolitiques. La perception qu'ont les acteurs les plus importants de l'extension et des éléments constitutifs de la crise peut influencer de façon significative leurs réactions, leurs attitudes et par conséquent l'issue du conflit. Mon point de vue est que l'on peut affiner l'instrument analytique en incorporant cet aspect de perception des tensions à la typologie d'Acuna - Smith sur les réponses démocratiques à des tensions sociopolitiques croissantes. Dans une perspective comparative, le niveau global des tensions sociopolitiques a été relativement bas dans toute l'Europe Centrale et de l'Est, y compris en Hongrie durant toute la période de transition ${ }^{48}$. Lorsque les tensions se sont accrues à l'automne 92 en Hongrie, elles n'ont certainement pas atteint des niveaux que le gouvernement Antall n'aurait pu neutraliser autrement que par cette convention sociale très démonstrative. En effet, plutôt que d'avoir recours à ses pratiques antérieures, le gouvernement réussit à calmer les tensions par des persuasions au cas par cas, des compensations partielles, "transactions"[[Concernant ces catégories, voir J. M. Nelson, "Overview. The Politics of Long-Haul Economic Reform" in J. M. Nelson (éd), Fragile Coalitions : The Politics of Economic Adjustment. Transaction Books. New Brunswick (USA) et Londres, 1989.], et en instaurant des limites. Pourtant, la perception du gouvernement du degré élevé des tensions, et son engagement dans la résolution ne changeait pas. Pourquoi ? Parce que, comme je l'ai déjà montré, leur perception de la crise était de nature essentiellement politique. Les inquiétudes modestes aux éruptions sporadiques de contestation sociale, prirent des proportions démesurées dès lors que la contestation a coïncidé avec la menace des votes de protestation au Parlement, des divisions dans la coalition et dans son parti le plus important. Ces inquiétudes augmentèrent également avec le souvenir de l'attitude de l'opposition à l'automne 1990 face au blocus des chauffeurs de taxi et à la crise sociopolitique. Comme le montre la popularité de la tentative de référendum, les craintes du gouvernement face à un possible accroissement de l'instabilité politique n'étaient pas vraiment injustifiées. Troisièmement, alors qu'Acuna et Smith centraient leur analyse sur l'attitude respective du gouvernement démocratique et de l'Etat comme élément explicatif majeur de la combinaison et du partage des moments d'inclusion et d'exclusion d'un régime démocratique, l'exemple hongrois montre que ce n'est pas toujours et nécessairement le gouvernement seul, qui décide du partage entre les exclus et les inclus, ni la manière dont cela se fait. En 1992, en Hongrie, l'incorporation des revendications des grévistes de la faim aux mesures de 
compensation ne s'est pas entièrement faite contre la volonté du gouvernement. Ce n'était pas non plus le gouvernement qui avait choisi le CRI comme plateforme des négociations, et qui par conséquent avait exclu la LAÉT et ses associés. Ce sont plutôt les syndicats qui ont fait le "sale boulot" de l'exclusion. Dans le cas du référendum, où le gouvernement aurait difficilement pu prévoir ce qui serait arrivé, et ce sont les partis politiques qui ont exclu leurs rivaux. C'est pourquoi le cas étudié ici peut constituer un apport dans l'élaboration et la définition plus juste du concept de "régime démocratique dual". S'il s'agit de comprendre les motifs, les mécanismes et les différents partages, et modèles possibles de combinaison entre exclusion et inclusion dans un régime démocratique dual, il peut s'avérer indispensable de combiner les explications axées sur l'Etat, avec celles axées sur la société. Ces dernières s'attachent à observer les processus relativement autonomes, les conditions, les combats et leurs conséquences sur le développement de la société civile et sur le type de démocratie que se met en place. Si un régime s'engage sur la voie de la démocratie (même s'il s'agit d'une démocratie duale), les élites à la tête de l'Etat auront à décider conjointement avec ceux qu'ils auront choisi pour partager le pouvoir, des moyens, du ratio, des méthodes et des action d'exclusion qu'ils devront employer.

\section{NOTES}

1. Une version préliminaire de cet article a été présentée à la conférence d'Essex sur la "Modernisation démocratique dans les pays d'Europe centrale », University of Essex, 11-14 mai 1994. Je remercie particulièrement Emil J. Kirchner et George Kolankiewicz pour leur précieux commentaires sur cette première version.

2. A. Gerschenkron, "Reflections on the Concept of « Prerequisites » of Modern Industrialisation", in Economic Backwardness in Historical Perspective, The Belknap Press of Harvard University Press, 1962.

3. G. O'Donnell, "On the State, Democratization and some Conceptual Problems (A Latin American View with Glances at some Post-Communist Countries)", World Development, Vol. 21, n 8, août, 1993. Pour une critique d'une téléologie de la transition, voir aussi M. Dobry, "Problems and Illusions in the Study of Transition to Democracy", Sisyphus, Social Studies. IFiS Publishers, IPS Publishers, Warsaw, 1992.

4. Voir les excellentes analyses de G. O'Donnell, "Delegative democracy ?", The helen Kellog Institute for International Studies, Working paper n 172, 1992 ; Acuna (C. H.), Smith (C. W.), "The Political Economy of Structural Adjustment : The Logic of Support and Opposition to Neoliberal Reform", in William C. Smith, Carlos H. Acuna and A. Camarra Eduardo (eds), Latin American Political Economy in the Ago of Neoliberal Reform. Theoritical and Comparative Perspectives for the 1990s, Transaction Publishers, New Brunswick (USA) et London (UK), 1994 ; N. Lechner, "Market Society and the Changing Pattern of Politics", texte présenté au XVIe Congrès Mondial de l'Intenational Political Science Association, août 21-25, 1994. Berlin mimeo.

5. I. Csurka, "Néhány gondolat... " ("Quelques pensées..."), Magyar fórum, 20 août, 1992. 
6. Le déficit supposé avoisinait les 180 à 200 milliards HUF, bien plus que les 70 milliards prévus. Le budget avait perdu environ 134 milliards de revenus, par rapport à l'année précedente, tandis que les dépenses restaient très élevées.

7. Le système hongrois de TVA., introduit en 1988, reposait sur trois niveaux : $0 \%, 15 \%$ et $25 \%$, affectant respectivement environ $44 \%, 5 \%$ et $45 \%$ de la consommation. Certains biens (liés principalement à la santé, la culture, l'éducation, etc.) étaient excemptés d'impôt.

8. Initiallement la grève de la faim était suivie avec une certaine réserve par la presse, elle a du même affronté l'hostilité de certains éléments de la presse écrite.

9. L. Vas, "Europeanisation and Interest groups in the New Hungarian Political System", Budapest Papers on Democratic Transition, no. 31. Le centre hongrois des études démocratiques, Département des sciences politiques, Budapest University of Economics. mimeo.

10. La confusion de la répresentation provenait de l'ampleur de l'émergence de plusieurs organisations écran dans le secteur civil, composées de membres extrêmement héterogénes et dotées une légitimitée très douteuse. Une d'entre elles était la Chambre sociale, considérée comme un des soutiens des grèves de la faim, et qui a fréquemment formulé des demandes, des revendications et des déclarations en leur nom. L. Herzog, "Experiences with Interest Reconciliation, and its Development", mimeo., octobre 1993.

11. Avec de maigres résultats, probablement. En effet, des factures téléphoniques après la grève de la faim, qui s'élévaient à cent mille forints (environ mille dollars américains) représentaient une dette scandaleuse pour la LAÉT (Magyar Hírlap, le 3 mars 1993).

12. Magyar Hírlap, le 31 octobre 1992.

13. Estimations d'experts que nous trouvont plus proches de la vérité que d'autres estimations.

14. Gy. Sziráczki, J. Szalai, M. Ladó, "Labour market trends and policies : Hungary" in G. Fischer et G. Standing (eds.) : Structural Change in Central and Eastern Europe : Labour Market and Social Policy Implications, OCDE, 1993.

15. Gy. Sziráczki, J. Szalai, M. Ladó, "Labour market trends and policies : Hungary" in G. Fischer et G. Standing (eds.) : Structural Change in Central and Eastern Europe : Labour Market and Social Policy Implications, OCDE, 1993.

16. L'éditorialiste du journal libéral caractérisa la situation de l'automne 1992 où culmina la grève de la faim du plus sérieux défi posé au gouvernement et à la démocratie hongroise depuis la grève des taxis de 1990. Magyar Hírlap, 8 novembre.

17. Gy. Sziráczki, J. Szalai, et M. Ladó, "Labour market trends and policies : Hungary" in G. Fischer et G. Standing (eds.), Structural Change in Central and Eastern Europe : Labour Market and Social Policy Implications, OCDE, 1993. p. 127.

18. K. Köhegyi, "A szocialis paktum politikai veszélyeiröl", Pénzugykutato Rt. (mimeo), 1994.

19. Magyar Hírlap, 3 novembre 1992.

20. Magyar Hírlap, 23 novembre 1992.

21. L. Héthy,"Tripartizmus és politikaformálás Magyarországon. Az 1992. novemberi ÉT magálla podás és összefüggései" (Tripartism, and the Formation of Politics in Hungary. The CRI-aggreement of November 1992, and its context). Munkaügyi Kutatóintézet és Magyar Munkaügyi Kapcsolatok Társaság - a Nemzetközi Szakértöi Kerekasztal Tanácskozás. Budapest, 1994. május 26-27. mimeo. 
22. Comme l'a affirmé le chef de Solidarité, Sándor Bátonyi : Les autres six syndicats n'étaient auparavant pas capables de faire accepter leur accord illégal, et le gouvernement ne voilait pas accepter leur proposition... Les négociations du CRI étaient utilisées pour faire chanter le gouvernement, qui était obligé d'accepter l'accord syndical. Voilà pourquoi les syndicats étaient si déterminés et intéressés à conserver tous les problèmes dans ensemble indivisible... Les citoyens ne seront pas contents des maigres résultats obtenus". (Magyar Hírlap du 23 novembre 1992). 23. Voir l'analyse pertinente du Ministre des Finances, Kupa, "The road to the agreement - or how much is that much ?", Magyar Hírlap, du 30 novembre 1992.

24. Magyar Hírlap du 8 décembre 1992.

25. Selon un sondage de fin 1992 , environ $25 \%$ des membres des partis de la coalition, et 40-50\% des électeurs de l'opposition, souhaitaient l'organisation d'élections générales plus tôt que prévu.

26. Magyar Hírlap du 7 décembre 1992.

27. Sur la carence de légitimité du régime hongrois voir János Kis, "Gondolatok a közeljövöröl" (Pensées sur le futur proche), in Magyar Hírlap du 24 décembre, 1992.

28. La MSZOSZ les rejoignit, déclarant son opposition à la dissolution du Parlement, et sa préférence pour des moyens corporatistes pour la résolution des tensions sociales. Magyar Hírlap du 8 janvier.

29. D'après Samuel Valenzuela : "Aucun leadership syndical rationel ne peut, par exemple, vouloir apporter son aide dans la lutte contre l'inflation si l'Etat lui-même n'est pas capable de limiter ses propres dépenses". Voir Samuel Valenzuela, "Labour Movements and Political Systems, A Conceptual and Typological Analysis", The Helen Kellogg Institute for International Studies. Working Paper No. 185.

30. Idem. Voir aussi O'Donnel et Schmitter : "S'il y un enseignement à tirer des efforts analogues menés par des démocraties politiques, en vue de poursuivre des politiques néocorporatistes concernant l'inflation, c'est que le succès dépend de la présence d'une association de classe autoritaire, monopolistique et centralisée forte d'un consensus solide sur les enjeux macro-économiques", in Transitions from Authoritarian Rule. Tentative Conclusions about Uncertain Democracies, The Johns Hopkins University Press, Baltimore and London, 1986, p. 46. Selon H. C. Acuna et C. W. Smith : « Les trois conditions préalables au succès de cette alternative sont : 1.) Un syndicat par classe capable d'obliger ses membres à se soumettre aux accords signés, 2.) Une grande institutionnalisation des relations capital - travail et les ouvriers et une capacité gouvernementale à sanctionner les violations de l'accord, et 3.) Peu de risques et d'incertitude pour l'investissement nécessaire à la baisse du chômage, à l'augmentation acceptable des salaires et à une croissance économique longue ", cf. "The Political Economy of Structural Adjustment : The Logic of Support and Opposition to Neoliberal Reform", in William C. Smith, Carlos H. Acuna et A. Camarra Eduardo (eds), Latin American Political Economy in the Ago of Neoliberal Reform. Theoritical and Comparative Perspectives for the 1990s, Transaction Publishers, New Brunswick (USA) et London (UK), 1994, p. 44. Voir aussi I. Roxborough : "Inflation and Social Pacts in Brazil and Mexico", Journal of Latin American Studies, vol. 24, part. 3, octobre 1992. 31. L. Héthy, op. cit.

32. C'est l'aspect sur lequel Adam Przeworski met l'accent lorsqu'il évalue la force économique relative du monde des affaires et des travailleurs comme facteur déterminant dans la conduite d'une concertation ou dans son échec. Voir "Capitalism, Democracy, Pacts : Revisited", la Conférence sur les micro-fondations de la démocratie, 
Université de Chicago du 29 avril au 1er mai, 1988, mimeo. Ce qui m'intéresse ici, plus précisement c'est la force politique relative du gouvernement et des syndicats.

33. Ce n'était pas la première fois dans l'histoire, puisque le pacte espagnol Moncloa a été conclu entre un gouvernement faible et les partis de l'oppostion, et soutenu mollement par un syndicat d'ouvriers faible qui ne l'a cependant pas signé.

34. Comme il l'avait fait une année plus tôt seulement, en 1991, lorsque l'un des organisateurs d'une grève préventive des cheminots avait été ostensiblement arrêté par la police, cinq minutes seulement avant la fin de la grève.

35. Voir par exemple J. Hausner, "Imperative vs. Interactive Strategy of Systemic Change in Central and Eastern Eurpe", 1993, et "Populist Threat in Transformation of Socialist Society, 1992 ; D. Ost : "Labor and Societal Transition", in Melvin Croan, Thomas E. Skidmore, David Ost, Lawrence S. Graham, Eric Hershberg, Is Latin America the Future of Eastern Europe ?, Problems of Communism, mai-juin, 1992. Voir aussi J. Nelson, "The Politics of Economic Transformation : Is Third World Experience Relevant in Eastern Europe ?", Overseas Development Council, mimeo, 1992.

36. Voir les exellentes études de C. Graham, "The vocal versus the Needy in Senegal : The Politics of Poverty Alleviation During Adjustment", 1992, "The Politics of Protecting the Poor During Adjustment : Bolivia's Emergency Social Fund", 1992, et "The Politics of Adjustment and Poverty in Zambia : The Hour Has Come !", 1992. 37. Voir notamment les excellentes analyses de Graham C. "The vocal versus the Needy in Senegal : The Politics of Poverty alleviation During Adjustment", The Brookings Institution, mimeo, 1992 ; "The Politics of Adjusment and Poverty in Zambia : The Hour has Come !", The Brookings Institution, mimeo, 1992 ; "The Politics of Protecting the Poor During Adjustment : Bolivia's Emergency Social Fund", World development, september 1992.

38. La formulation est d'o Donnel et Schmitter.

39. Métaphore utilisée pour démontrer le niveau d'incertitude dans un processus de changement systémique. Bunce V. et Csanadi M « the systematic analysis of a non system » Gyorgy Szoboszlai Flying blind. Emerging democracies in East-central Europe Yearbook of the hungarian political science association 1992.

40. Voici des questions formulées par O'Donnell, Acuna, Smith et autres théoriciens dans leur conceptualisation de nouveaux types de démocratie.

41. La démocratie duale se produira le plus plausiblement dans la plupart des sociétés latino-américaines, selon Acuna et Smith. Voir H. C. Acuna et C. W. Smith, op. cit., p. 53. 42. H. C. Acuna et C. W. Smith, op. cit., p. 47.

43. Idem, p. 53.

44. Il est certain que la solution du conflit autour du réferendum manqué était tout autant de nature élitiste, poursuivant la tradition élitiste des autres accords de transition : le pacte MDF-SZDSZ, par exemple, duquel tous les autres partis du Parlement, qu'ils soient de l'opposition ou de la majorité, avaient été exclus. A l'évidence, la notion "élitiste" ne signifie en général pas "antidémocratique" dans ce contexte, mais indique plutôt des moyens spécifiques pour consolider la démocratie, ainsi que les caractéristiques principales du régime en évolution. Des accords aussi élitistes pour consolider le système ont eu lieu après les élections du printemps 1994, ainsi qu'entre les partenaires de la coalition pour redéfinir les règles du jeu.

45. László Bruszt, ("Le gouvernement Antall et les associations d'intérêt économiques"), in Kormány a mérlegen 1990-1994, Politikai Kutatások Központja, Budapest, Korridor, 1994. 
46. H. C. Acuna et C. W. Smith : op. cit., p. 48.

47. M.S. Grindle et J. W. Thomas : Public Choices and Policy Change. The Political Economy of Reform in Developing Countries, The John Hopkins University Press, Baltimore and London, 1991.

48. Voir quelques explications possibles chez B. Greskovits, "Is the East Becoming the South? Where Threats to Reforms May Come From ?", texte présenté au XVIe Congrès Mondial de l'Intenational Political Science Association, août 21-25, 1994. Berlin mimeo.

\section{INDEX}

Mots-clés : démocratie, mobilisation, mouvements politiques, Mouvements sociaux

Index géographique : Europe centrale et orientale

Index chronologique : 1989

\section{AUTEUR}

\section{BÉLA GRESKOVITS}

Professeur au département des sciences politiques de l'Université d'Europe Centrale à Budapest. 